Copyright (C 2015 IEEE. Personal use of this material is permitted. Permission from IEEE must be obtained for all other uses, in any current or future media, including reprinting/republishing this material for advertising or promotional purposes, creating new collective works, for resale or redistribution to servers or lists, or reuse of any copyrighted component of this work in other works. 


\section{Transceiver Optimization for Interference MIMO Relay Systems Using the Structure of Relay Matrix}

\author{
Khoa Xuan Nguyen \\ Dept. Electrical and Computer Eng. \\ Curtin University \\ Bentley, WA, 6102, Australia \\ khoa.x.nguyen@ieee.org
}

\author{
Yue Rong \\ Dept. Electrical and Computer Eng. \\ Curtin University \\ Bentley, WA, 6102, Australia \\ y.rong@curtin.edu.au
}

\author{
Sven Nordholm \\ Dept. Electrical and Computer Eng. \\ Curtin University \\ Bentley, WA, 6102, Australia \\ s.nordholm@curtin.edu.au
}

\begin{abstract}
In this paper, we study the transceiver design problem for amplify-and-forward interference multiple-input multiple-output (MIMO) relay communication systems, where multiple transmitter-receiver pairs communicate simultaneously with the aid of a relay node. We aim at minimizing the mean-squared error (MSE) of the signal waveform estimation at the receivers subjecting to transmission power constraints at the transmitters and the relay node. Since the transceiver optimization problem is nonconvex with matrix variables, the globally optimal solution is intractable to obtain. To overcome the challenge, we propose an iterative transceiver design algorithm where the transmitter, relay, and receiver matrices are optimized iteratively by exploiting the optimal structure of the relay precoding matrix. Simulation results show that the proposed algorithm performs better than the existing technique in terms of both MSE and bit-error-rate.

Index Terms-Interference channel, MIMO relay, MSE
\end{abstract}

\section{INTRODUCTION}

Multiple-input multiple-output (MIMO) relay communications have attracted much research interest recently [1], [2]. In MIMO relay systems, communication between source and destination nodes is assisted by single or multiple relays equipped with multiple antennas. The relay nodes can either decode-and-forward (DF) or amplify-and-forward (AF) the relayed signals [3]. In the AF protocol, the signals received at the relay nodes are simply amplified (including a possible linear transformation) through the relay precoding matrices before being forwarded to the destination nodes. Therefore, in general the AF protocol has lower complexity and shorter processing delay than the DF strategy. For single-user twohop AF MIMO relay communication systems, the optimal source and relay precoding matrices have been developed in [4]-[6]. Recent progress on the optimization of AF MIMO relay systems has been summarized in [2].

For MIMO interference channels, the idea of interference alignment (IA) [7] has been developed for interference suppression by arranging the desired signal and interference into corresponding signal spaces. The idea of IA has been applied to interference MIMO relay systems in [8], [9]. In [10], an iterative algorithm has been proposed to optimize the source beamforming vector and the relay precoding matrices to minimize the total source and relay transmit power such that a minimum signal-to-interference-plus-noise ratio
(SINR) threshold is maintained at each receiver. An iterative transceiver design algorithm has been developed in [11] to minimize the total interference in interference MIMO relay systems.

In this paper, we consider an interference MIMO relay system where multiple transmitter-receiver pairs communicate simultaneously with the aid of a relay node. The transmitters, receivers, and the relay node are equipped with multiple antennas. Since the raw bit-error-rate (BER) is closely related to the mean-squared error (MSE) of the signal waveform estimation at the receivers, the minimal MSE (MMSE) is chosen as the design criterion. We aim at optimizing the transmitter, relay, and receiver matrices to suppress the interference and minimize the sum MSE (SMSE) of the signal waveform estimation at the receivers, subjecting to transmission power constraints at transmitters and the relay node.

Since the transceiver optimization problem is nonconvex with matrix variables, a globally optimal solution is computationally intractable to obtain. To overcome the challenge, we propose an iterative transceiver design algorithm through solving convex subproblems. In each iteration of this algorithm, we first update the receiver matrices based on the transmitter and relay matrices from the previous iteration. Then we optimize the relay matrix based on its optimal structure, the transmitter matrices from the previous iteration, and the receiver matrices in this iteration. Finally, the transmitter matrices are updated. The MSE and bit-error-rate (BER) simulation results demonstrate that the proposed algorithm converges in a few iterations.

\section{System Model And Problem Formulation}

We consider a two-hop interference MIMO relay communication system where $K$ transmitter-receiver pairs communicate simultaneously with the aid of a single relay node as shown in Fig.1. For simplicity, the direct links between transmitters and receivers are ignored as they undergo much larger path attenuation compared with the links via the relay node [10]. The $k$ th transmitter and receiver are equipped with $N_{s k}$ and $N_{d k}$ antennas, respectively, and the number of antennas at the relay node is $N_{r}$.

The communication between transmitter-receiver pairs is completed in two time slots. In the first time slot, the $k$ th transmitter encodes the $d \times 1$ information-carrying symbol 


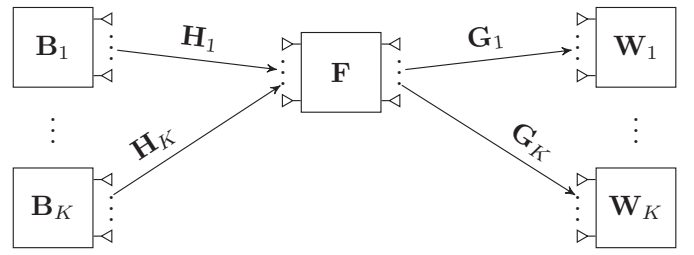

Fig. 1. Block diagram of an interference MIMO relay system.

vector $\mathbf{s}_{k}$ with the $N_{s k} \times d$ transmitter precoding matrix $\mathbf{B}_{k}$ before transmitting the $N_{s k} \times 1$ precoded signal vector

$$
\mathbf{x}_{s k}=\mathbf{B}_{k} \mathbf{s}_{k}, \quad k=1, \cdots, K
$$

to the relay node. The received signal vector at the relay node is given by

$$
\mathbf{y}_{r}=\sum_{k=1}^{K} \mathbf{H}_{k} \mathbf{B}_{k} \mathbf{s}_{k}+\mathbf{v}_{r}
$$

where $\mathbf{H}_{k}$ is the $N_{r} \times N_{s k}$ MIMO channel matrix between the $k$ th transmitter and the relay node, $\mathbf{v}_{r}$ is the $N_{r} \times 1$ additive white Gaussian noise (AWGN) vector at the relay node with zero mean and covariance matrix $E\left[\mathbf{v}_{r} \mathbf{v}_{r}^{H}\right]=\sigma_{r}^{2} \mathbf{I}_{N_{r}}$. Here $(\cdot)^{H}$ denote matrix conjugate transpose, $E[\cdot]$ denotes the statistical expectation, and $\mathbf{I}_{n}$ stands for the $n \times n$ identity matrix.

In the second time slot, the relay node amplifies the received signal vector with the $N_{r} \times N_{r}$ precoding matrix $\mathbf{F}$ as

$$
\mathbf{x}_{r}=\mathbf{F} \mathbf{y}_{r} \text {. }
$$

The precoded signal vector $\mathbf{x}_{r}$ is forwarded to the receivers. The received signal vector at the $k$ th receiver is given by

$$
\mathbf{y}_{d k}=\mathbf{G}_{k} \mathbf{x}_{r}+\mathbf{v}_{d k}, \quad k=1, \cdots, K
$$

where $\mathbf{G}_{k}$ is the $N_{d k} \times N_{r}$ MIMO channel matrix between the relay node and the $k$ th receiver, $\mathbf{v}_{d k}$ is the $N_{d k} \times 1$ AWGN vector at the $k$ th receiver with zero mean and covariance matrix $E\left[\mathbf{v}_{d k} \mathbf{v}_{d k}^{H}\right]=\sigma_{d k}^{2} \mathbf{I}_{N_{d k}}$.

Due to their simplicity, linear receivers are used to retrieve the transmitted signals, and we have $d \leq N_{r}$ and $d \leq N_{d k}$, $k=1, \cdots, K$. The estimated signal vector at the $k$ th receiver can be written as

$$
\hat{\mathbf{s}}_{k}=\mathbf{W}_{k}^{H} \mathbf{y}_{d k}, \quad k=1, \ldots, K
$$

where $\mathbf{W}_{k}$ is the $N_{d k} \times d$ receiver weight matrix. Using (2)(4), the estimated signal vector in (5) becomes

$$
\begin{aligned}
\hat{\mathbf{s}}_{k} & =\underbrace{\mathbf{W}_{k}^{H}\left(\mathbf{G}_{k} \mathbf{F} \sum_{m=1}^{K} \mathbf{H}_{m} \mathbf{B}_{m} \mathbf{s}_{m}+\overline{\mathbf{v}}_{d k}\right)}_{\text {desired signal }} \\
& =\underbrace{\mathbf{W}_{k}^{H} \mathbf{G}_{k} \mathbf{F} \mathbf{H}_{k} \mathbf{B}_{k} \mathbf{s}_{k}}_{\text {interference plus noise }}
\end{aligned}
$$

where $\overline{\mathbf{v}}_{d k} \triangleq \mathbf{G}_{k} \mathbf{F} \mathbf{v}_{r}+\mathbf{v}_{d k}$ is the total noise vector at the $k$ th receiver.

The signal vectors sent by transmitters and the signal vector forwarded from the relay node must satisfy the following transmission power constraints

$$
\begin{aligned}
& \operatorname{tr}\left(\mathbf{B}_{k} E\left[\mathbf{s}_{k} \mathbf{s}_{k}^{H}\right] \mathbf{B}_{k}^{H}\right) \leq P_{s k}, \quad k=1, \cdots, K \\
& \operatorname{tr}\left(\mathbf{F} E\left[\mathbf{y}_{r} \mathbf{y}_{r}^{H}\right] \mathbf{F}^{H}\right) \leq P_{r}
\end{aligned}
$$

where $\operatorname{tr}(\cdot)$ stands for matrix trace, $P_{s k}$ and $P_{r}$ denote the power budget at the $k$ th transmitter and the relay node, respectively, $E\left[\mathbf{s}_{k} \mathbf{s}_{k}^{H}\right]=\mathbf{I}_{d}$ is the covariance matrix of the information-carrying symbol vector at the $k$ th transmitter, and $E\left[\mathbf{y}_{r} \mathbf{y}_{r}^{H}\right]=\sum_{m=1}^{K} \mathbf{H}_{m} \mathbf{B}_{m} \mathbf{B}_{m}^{H} \mathbf{H}_{m}^{H}+\sigma_{r}^{2} \mathbf{I}_{N_{r}}$ is the covariance matrix of the received signal vector at the relay node.

From (6), the MSE of estimating $\mathbf{s}_{k}$ can be calculated as

$$
\begin{aligned}
\operatorname{MSE}_{k}= & \operatorname{tr}\left(E\left[\left(\hat{\mathbf{s}}_{k}-\mathbf{s}_{k}\right)\left(\hat{\mathbf{s}}_{k}-\mathbf{s}_{k}\right)^{H}\right]\right) \\
= & \operatorname{tr}\left(\left(\mathbf{W}_{k}^{H} \mathbf{L}_{k}-\mathbf{I}_{d}\right)\left(\mathbf{W}_{k}^{H} \mathbf{L}_{k}-\mathbf{I}_{d}\right)^{H}\right. \\
& \left.+\mathbf{W}_{k}^{H} \mathbf{C}_{n k} \mathbf{W}_{k}+\mathbf{W}_{k}^{H} \boldsymbol{\Xi}_{k} \mathbf{W}_{k}\right), k=1, \cdots, K
\end{aligned}
$$

where $\mathbf{L}_{k}$ is the equivalent MIMO channel matrix of the $k$ th transmitter-receiver pair, $\mathbf{C}_{n k}=E\left[\overline{\mathbf{v}}_{d k} \overline{\mathbf{v}}_{d k}^{H}\right]$ is the covariance matrix of the equivalent noise, and $\boldsymbol{\Xi}_{k}$ is the covariance matrix of interference at the $k$ th receiver. They are given respectively as

$$
\begin{aligned}
\mathbf{L}_{k} & =\mathbf{G}_{k} \mathbf{F} \overline{\mathbf{H}}_{k}, \quad k=1, \cdots, K \\
\mathbf{C}_{n k} & =E\left[\left(\mathbf{G}_{k} \mathbf{F} \mathbf{v}_{r}+\mathbf{v}_{d k}\right)\left(\mathbf{G}_{k} \mathbf{F} \mathbf{v}_{r}+\mathbf{v}_{d k}\right)^{H}\right] \\
& =\sigma_{r}^{2} \mathbf{G}_{k} \mathbf{F} \mathbf{F}^{H} \mathbf{G}_{k}^{H}+\sigma_{d k}^{2} \mathbf{I}_{N_{d k}}, \quad k=1, \cdots, K \\
\mathbf{\Xi}_{k} & =\mathbf{G}_{k} \mathbf{F} \sum_{m=1, m \neq k}^{K} \overline{\mathbf{H}}_{m} \overline{\mathbf{H}}_{m}^{H} \mathbf{F}^{H} \mathbf{G}_{k}^{H}, \quad k=1, \cdots, K
\end{aligned}
$$

where $\overline{\mathbf{H}}_{k} \triangleq \mathbf{H}_{k} \mathbf{B}_{k}$ is the equivalent MIMO channel matrix between the $k$ th transmitter and the relay node.

We aim at optimizing the transmitter precoding matrices $\left\{\mathbf{B}_{k}\right\} \triangleq\left\{\mathbf{B}_{k}, k=1, \cdots, K\right\}$, the relay precoding matrix $\mathbf{F}$, and the receiver weight matrices $\left\{\mathbf{W}_{k}\right\} \triangleq\left\{\mathbf{W}_{k}, k=\right.$ $1, \cdots, K\}$, to minimize the sum-MSE of the signal waveform estimation at the receivers under transmission power constraints at the transmitters and the relay node. From (7)(9), the optimal transmitter, relay, and receiver matrices design problem can be written as

$$
\begin{aligned}
\min _{\left\{\mathbf{W}_{k}\right\},\left\{\mathbf{B}_{k}\right\}, \mathbf{F}} & \sum_{k=1}^{K} \mathrm{MSE}_{k} \\
\text { s.t. } & \operatorname{tr}\left(\mathbf{B}_{k} \mathbf{B}_{k}^{H}\right) \leq P_{s k}, \quad k=1, \cdots, K \\
& \operatorname{tr}\left(\mathbf{F} E\left[\mathbf{y}_{r} \mathbf{y}_{r}^{H}\right] \mathbf{F}^{H}\right) \leq P_{r} .
\end{aligned}
$$

\section{Proposed Transmitter, Relay, And Receiver MATRICES Design Algorithm}

The problem (10)-(12) is highly nonconvex with matrix variables, and a globally optimal solution is intractable to obtain. To overcome this challenge, we propose an iterative 
algorithm to solve the problem (10)-(12) by optimizing $\left\{\mathbf{W}_{k}\right\}$, $\left\{\mathbf{B}_{k}\right\}$, and $\mathbf{F}$ in an alternating way through solving convex subproblems.

It can be seen from (7) and (8) that the power constraints are independent of $\left\{\mathbf{W}_{k}\right\}$. Thus, with given relay matrix and transmitter matrices, the optimal linear receiver matrix which minimizes MSE in (9) is the well-known MMSE receiver

$$
\mathbf{W}_{k}=\left(\mathbf{L}_{k} \mathbf{L}_{k}^{H}+\mathbf{C}_{n k}+\mathbf{\Xi}_{k}\right)^{-1} \mathbf{L}_{k}, \quad k=1, \cdots, K
$$

where $(\cdot)^{-1}$ denotes matrix inverse.

With given transmitter matrices $\left\{\mathbf{B}_{k}\right\}$ and receiver matrices $\left\{\mathbf{W}_{k}\right\}$ obtained in (13), the sum-MSE SMSE $=\sum_{k=1}^{K} \mathrm{MSE}_{k}$ can be rewritten as a function of $\mathbf{F}$ as

$$
\begin{aligned}
\mathrm{SMSE}= & \sum_{k=1}^{K} \operatorname{tr}\left(\left(\mathbf{W}_{k}^{H} \mathbf{G}_{k} \mathbf{F} \overline{\mathbf{H}}_{k}-\mathbf{I}_{d}\right)\left(\mathbf{W}_{k}^{H} \mathbf{G}_{k} \mathbf{F} \overline{\mathbf{H}}_{k}-\mathbf{I}_{d}\right)^{H}\right. \\
& +\sigma_{r}^{2} \mathbf{W}_{k}^{H} \mathbf{G}_{k} \mathbf{F} \mathbf{F}^{H} \mathbf{G}_{k}^{H} \mathbf{W}_{k}+\sigma_{d k}^{2} \mathbf{W}_{k}^{H} \mathbf{W}_{k} \\
& \left.+\mathbf{W}_{k}^{H} \mathbf{G}_{k} \mathbf{F} \sum_{m=1, m \neq k}^{K} \overline{\mathbf{H}}_{m} \overline{\mathbf{H}}_{m}^{H} \mathbf{F}^{H} \mathbf{G}_{k}^{H} \mathbf{W}_{k}\right) .
\end{aligned}
$$

Let us introduce

$$
\begin{aligned}
& \mathbf{H}=\left[\mathbf{H}_{1} \mathbf{B}_{1}, \ldots, \mathbf{H}_{K} \mathbf{B}_{K}\right]=\mathbf{U}_{h} \boldsymbol{\Lambda}_{h} \mathbf{V}_{h}^{H} \\
& \mathbf{G}=\left[\mathbf{G}_{1}^{T}, \ldots, \mathbf{G}_{K}^{T}\right]^{T}=\mathbf{U}_{g} \boldsymbol{\Lambda}_{g} \mathbf{V}_{g}^{H}
\end{aligned}
$$

as the singular-value decomposition (SVD) of the equivalent transmitters-relay channel $\mathbf{H}$ and the equivalent relay-receivers channel G, where $(\cdot)^{T}$ denotes matrix transpose. The dimensions of $\mathbf{U}_{h}, \boldsymbol{\Lambda}_{h}, \mathbf{V}_{h}$ are $N_{r} \times L_{1}, L_{1} \times L_{1}, K d \times L_{1}$, respectively and the dimensions of $\mathbf{U}_{g}, \boldsymbol{\Lambda}_{g}, \mathbf{V}_{g}$ are $\bar{N}_{d} \times L_{2}$, $L_{2} \times L_{2}, N_{r} \times L_{2}$, respectively, where $\bar{N}_{d} \triangleq \sum_{k=1}^{K} N_{d k}$, $L_{1} \triangleq \min \left(K d, N_{r}\right)$, and $L_{2} \triangleq \min \left(\bar{N}_{d}, N_{r}\right)$.

It can be shown similar to [12] that the optimal structure of the relay precoding matrix $\mathbf{F}$ is

$$
\mathbf{F}=\mathbf{V}_{g} \mathbf{A} \mathbf{U}_{h}^{H}
$$

where $\mathbf{A}$ is an $L_{2} \times L_{1}$ matrix. It can be seen from (17) that we only need to optimize $\mathbf{A}$ in order to optimize $\mathbf{F}$. Since the dimension of $\mathbf{A}$ is smaller than or equal to that of $\mathbf{F}$, optimizing A may have a smaller computational complexity than directly optimizing $\mathbf{F}$.

From (15) and (16), we have for $k=1, \cdots, K$

$$
\mathbf{H}_{k} \mathbf{B}_{k}=\mathbf{U}_{h} \boldsymbol{\Lambda}_{h} \mathbf{V}_{h, k}^{H}, \mathbf{G}_{k}=\mathbf{U}_{g, k} \boldsymbol{\Lambda}_{g} \mathbf{V}_{g}^{H}
$$

where $\mathbf{V}_{h, k}$ contains the $((k-1) d+1)$-th to the $k d$-th rows of $\mathbf{V}_{h}$, and $\mathbf{U}_{g, k}$ contains the $\left(\sum_{i=1}^{k-1} N_{d i}+1\right)$-th to the $\left(\sum_{i=1}^{k} N_{d i}\right)$-th rows of $\mathbf{U}_{g}$, that is, $\mathbf{V}_{h}=\left[\mathbf{V}_{h, 1}^{T}, \ldots, \mathbf{V}_{h, K}^{T}\right]^{T}$, $\mathbf{U}_{g}=\left[\mathbf{U}_{g, 1}^{T}, \ldots, \mathbf{U}_{g, K}^{T}\right]^{T}$. Note that $\mathbf{V}_{h, k}$ and $\mathbf{U}_{g, k}$ have dimensions of $d \times L_{1}$ and $N_{d k} \times L_{2}$, respectively. By substituting (17) back into (14), the SMSE becomes

$$
\begin{aligned}
& \mathrm{SMSE}=\sum_{k=1}^{K} \operatorname{tr}\left(\left(\mathbf{W}_{k}^{H} \mathbf{U}_{g, k} \boldsymbol{\Lambda}_{g} \mathbf{A} \mathbf{\Lambda}_{h} \mathbf{V}_{h, k}^{H}-\mathbf{I}_{d}\right)\right. \\
& \times\left(\mathbf{W}_{k}^{H} \mathbf{U}_{g, k} \boldsymbol{\Lambda}_{g} \mathbf{A} \boldsymbol{\Lambda}_{h} \mathbf{V}_{h, k}^{H}-\mathbf{I}_{d}\right)^{H}
\end{aligned}
$$

$$
\begin{aligned}
& +\mathbf{W}_{k}^{H} \mathbf{U}_{g, k} \boldsymbol{\Lambda}_{g} \mathbf{A} \sum_{m=1, m \neq k}^{K} \boldsymbol{\Lambda}_{h} \mathbf{V}_{h, m}^{H} \mathbf{V}_{h, m} \boldsymbol{\Lambda}_{h} \mathbf{A}^{H} \boldsymbol{\Lambda}_{g} \mathbf{U}_{g, k}^{H} \mathbf{W}_{k} \\
& \left.+\sigma_{r}^{2} \mathbf{W}_{k}^{H} \mathbf{U}_{g, k} \boldsymbol{\Lambda}_{g} \mathbf{A} \mathbf{A}^{H} \boldsymbol{\Lambda}_{g} \mathbf{U}_{g, k}^{H} \mathbf{W}_{k}+\sigma_{d k}^{2} \mathbf{W}_{k}^{H} \mathbf{W}_{k}\right) .
\end{aligned}
$$

Using the identities of [13]

$$
\begin{aligned}
\operatorname{tr}\left(\mathbf{A}^{T} \mathbf{B}\right) & =(\operatorname{vec}(\mathbf{A}))^{T} \operatorname{vec}(\mathbf{B}) \\
\operatorname{tr}\left(\mathbf{A}^{H} \mathbf{B} \mathbf{A C}\right) & =(\operatorname{vec}(\mathbf{A}))^{H}\left(\mathbf{C}^{T} \otimes \mathbf{B}\right) \operatorname{vec}(\mathbf{A}) \\
\operatorname{vec}(\mathbf{A B C}) & =\left(\mathbf{C}^{T} \otimes \mathbf{A}\right) \operatorname{vec}(\mathbf{B})
\end{aligned}
$$

where $\operatorname{vec}(\cdot)$ stacks columns of a matrix on top of each other into a single vector and $\otimes$ represents the Kronecker product, the SMSE (19) can be represented as a function of $\mathbf{a} \triangleq \operatorname{vec}(\mathbf{A})$ as

$$
\begin{aligned}
\mathrm{SMSE}= & \sum_{k=1}^{K}\left[\left(\mathbf{O}_{k} \mathbf{a}-\operatorname{vec}\left(\mathbf{I}_{d}\right)\right)^{H}\left(\mathbf{O}_{k} \mathbf{a}-\operatorname{vec}\left(\mathbf{I}_{d}\right)\right)\right. \\
& \left.+\mathbf{a}^{H} \mathbf{Q}_{k} \mathbf{a}+\mathbf{a}^{H} \mathbf{S}_{k} \mathbf{a}\right]+t_{1}
\end{aligned}
$$

where $t_{1} \triangleq \sum_{k=1}^{K} \sigma_{d k}^{2} \operatorname{tr}\left(\mathbf{W}_{k}^{H} \mathbf{W}_{k}\right)$ does not depend on $\mathbf{a}$, and for $k=1, \cdots, K$

$$
\begin{aligned}
\mathbf{O}_{k}= & \left(\boldsymbol{\Lambda}_{h} \mathbf{V}_{h, k}^{H}\right)^{T} \otimes\left(\mathbf{W}_{k}^{H} \mathbf{U}_{g, k} \boldsymbol{\Lambda}_{g}\right) \\
\mathbf{Q}_{k}= & \sigma_{r}^{2} \mathbf{I}_{L_{1}} \otimes\left(\boldsymbol{\Lambda}_{g} \mathbf{U}_{g, k}^{H} \mathbf{W}_{k} \mathbf{W}_{k}^{H} \mathbf{U}_{g, k} \boldsymbol{\Lambda}_{g}\right) \\
\mathbf{S}_{k}= & \left(\sum_{m=1, m \neq k}^{K} \boldsymbol{\Lambda}_{h} \mathbf{V}_{h, m}^{H} \mathbf{V}_{h, m} \boldsymbol{\Lambda}_{h}\right)^{T} \\
& \otimes\left(\boldsymbol{\Lambda}_{g} \mathbf{U}_{g, k}^{H} \mathbf{W}_{k} \mathbf{W}_{k}^{H} \mathbf{U}_{g, k} \boldsymbol{\Lambda}_{g}\right) .
\end{aligned}
$$

From (17), the relay node transmission power constraint (8) can be written as

$$
\operatorname{tr}\left(\mathbf{F} E\left[\mathbf{y}_{r} \mathbf{y}_{r}^{H}\right] \mathbf{F}^{H}\right)=\operatorname{tr}\left(\mathbf{A}\left(\boldsymbol{\Lambda}_{h}^{2}+\sigma_{r}^{2} \mathbf{I}_{L_{1}}\right) \mathbf{A}^{H}\right) .
$$

By introducing $\mathbf{D}=\left(\boldsymbol{\Lambda}_{h}^{2}+\sigma_{r}^{2} \mathbf{I}_{L_{1}}\right) \otimes \mathbf{I}_{L_{2}}$, (24) can be rewritten as

$$
\mathbf{a}^{H} \mathbf{D a} \leq P_{r} .
$$

From (23) and (25), the relay matrix optimization problem can be written as

$$
\begin{aligned}
& \min _{\mathbf{a}} \operatorname{SMSE} \\
& \text { s.t. } \mathbf{a}^{H} \mathbf{D a} \leq P_{r} .
\end{aligned}
$$

The problem (26)-(27) is a quadratically constrained quadratic programming (QCQP) problem [14], which is a convex optimization problem and can be efficiently solved by the interiorpoint method [14]. The problem (26)-(27) can be solved by the CVX MATLAB toolbox for disciplined convex programming [15].

With given receiver matrices $\left\{\mathbf{W}_{k}\right\}$ and the relay matrix $\mathbf{F}$, the sum-MSE can be rewritten as a function of $\left\{\mathbf{B}_{k}\right\}$ as

$$
\begin{aligned}
\mathrm{SMSE}= & \sum_{k=1}^{K} \operatorname{tr}\left(\left(\overline{\mathbf{G}}_{k} \mathbf{F} \mathbf{H}_{k} \mathbf{B}_{k}-\mathbf{I}_{d}\right)\left(\overline{\mathbf{G}}_{k} \mathbf{F} \mathbf{H}_{k} \mathbf{B}_{k}-\mathbf{I}_{d}\right)^{H}\right. \\
& \left.+\overline{\mathbf{G}}_{k} \mathbf{F} \sum_{m=1, m \neq k}^{K} \mathbf{H}_{m} \mathbf{B}_{m} \mathbf{B}_{m}^{H} \mathbf{H}_{m}^{H} \mathbf{F}^{H} \overline{\mathbf{G}}_{k}^{H}\right)+t_{2}
\end{aligned}
$$


where $\overline{\mathbf{G}}_{k}=\mathbf{W}_{k}^{H} \mathbf{G}_{k}$ and $t_{2} \triangleq \sum_{k=1}^{K} \operatorname{tr}\left(\mathbf{W}_{k}^{H} \mathbf{C}_{n k} \mathbf{W}_{k}\right)$ can be ignored in the optimization process as it does not depend on $\left\{\mathbf{B}_{k}\right\}$.

Using the identities in (20)-(22), the SMSE function in (28) can be written as a function of $\mathbf{b}_{k} \triangleq \operatorname{vec}\left(\mathbf{B}_{k}\right)$ as

SMSE

$$
\begin{aligned}
= & \sum_{k=1}^{K}\left[\left(\mathbf{S}_{k} \mathbf{b}_{k}-\operatorname{vec}\left(\mathbf{I}_{d}\right)\right)^{H}\left(\mathbf{S}_{k} \mathbf{b}_{k}-\operatorname{vec}\left(\mathbf{I}_{d}\right)\right)\right. \\
& \left.+\sum_{m=1, m \neq k}^{K} \mathbf{b}_{m}^{H}\left(\mathbf{I}_{d} \otimes \mathbf{H}_{m}^{H} \mathbf{F}^{H} \overline{\mathbf{G}}_{k}^{H} \overline{\mathbf{G}}_{k} \mathbf{F} \mathbf{H}_{m}\right) \mathbf{b}_{m}\right]+t_{2} \\
= & \sum_{k=1}^{K}\left[\left(\mathbf{S}_{k} \mathbf{b}_{k}-\operatorname{vec}\left(\mathbf{I}_{d}\right)\right)^{H}\left(\mathbf{S}_{k} \mathbf{b}_{k}-\operatorname{vec}\left(\mathbf{I}_{d}\right)\right)+\mathbf{b}_{k}^{H} \mathbf{T}_{k} \mathbf{b}_{k}\right]+t_{2}
\end{aligned}
$$

where for $k=1, \cdots, K, \mathbf{S}_{k} \triangleq \mathbf{I}_{d} \otimes\left(\overline{\mathbf{G}}_{k} \mathbf{F} \mathbf{H}_{k}\right), \mathbf{T}_{k} \triangleq \mathbf{I}_{d} \otimes$ $\sum_{m=1, m \neq k}^{K} \mathbf{H}_{k}^{H} \mathbf{F}^{H} \overline{\mathbf{G}}_{m}^{H} \overline{\mathbf{G}}_{m} \mathbf{F} \mathbf{H}_{k}$.

By introducing $\mathbf{T} \triangleq b d\left(\mathbf{T}_{1}, \cdots, \mathbf{T}_{K}\right)$, where $b d(\cdot)$ denotes a block-diagonal matrix, and $\overline{\mathbf{S}}_{k} \triangleq\left[\mathbf{S}_{k 1}, \cdots, \mathbf{S}_{k K}\right]$, where $\mathbf{S}_{k k}=\mathbf{S}_{k}$ and $\mathbf{S}_{k i}=\mathbf{0}, i \neq k$, the SMSE function (29) can be written as a function of $\mathbf{b}=\left[\mathbf{b}_{1}^{T}, \mathbf{b}_{2}^{T}, \cdots, \mathbf{b}_{K}^{T}\right]^{T}$ as

$$
\Phi_{1}(\mathbf{b})=\sum_{k=1}^{K}\left(\overline{\mathbf{S}}_{k} \mathbf{b}-\operatorname{vec}\left(\mathbf{I}_{d}\right)\right)^{H}\left(\overline{\mathbf{S}}_{k} \mathbf{b}-\operatorname{vec}\left(\mathbf{I}_{d}\right)\right)+\mathbf{b}^{H} \mathbf{T} \mathbf{b} .
$$

Let us introduce $\mathbf{E}_{j}=\mathbf{I}_{d} \otimes\left(\mathbf{H}_{j}^{H} \mathbf{F}^{H} \mathbf{F} \mathbf{H}_{j}\right), \mathbf{E}=$ $b d\left(\mathbf{E}_{1}, \mathbf{E}_{2}, \cdots, \mathbf{E}_{K}\right), \overline{\mathbf{E}}_{i}=b d\left(\overline{\mathbf{E}}_{i 1}, \overline{\mathbf{E}}_{i 2}, \cdots, \overline{\mathbf{E}}_{i K}\right)$, where $\overline{\mathbf{E}}_{i i}=\mathbf{I}_{d N_{s}}$ and $\overline{\mathbf{E}}_{i j}=\mathbf{0}, i \neq j$. The optimal $\mathbf{b}$ can be obtained by solving the following problem

$$
\begin{aligned}
\min _{\mathbf{b}} & \Phi_{1}(\mathbf{b}) \\
\text { s.t. } & \mathbf{b}^{H} \overline{\mathbf{E}}_{k} \mathbf{b} \leq P_{s k}, \quad k=1, \cdots, K \\
& \mathbf{b}^{H} \mathbf{E} \mathbf{b} \leq P_{r}-\sigma_{r}^{2} \operatorname{tr}\left(\mathbf{F F}^{H}\right) .
\end{aligned}
$$

The problem (31)-(33) is a QCQP problem and can be solved by the CVX MATLAB toolbox [15] for disciplined convex programming.

The steps of applying the proposed algorithm to optimize $\left\{\mathbf{B}_{k}\right\}, \mathbf{F}$, and $\left\{\mathbf{W}_{k}\right\}$ are summarized in Table I, where the superscript $(n)$ denotes the variable at the $n$th iteration, and $\varepsilon$ is a small positive number up to which convergence is acceptable. Since all subproblems (10), (26)-(27), and (31)(33) are convex, the solution to each subproblem is optimal. Thus, the conditional updates of $\left\{\mathbf{B}_{k}\right\}, \mathbf{F}$, and $\left\{\mathbf{W}_{k}\right\}$ may either decrease or maintain but cannot increase the objective function (10). Moreover, the objective function is lower bounded by at least zero. Therefore, the iterative algorithm converges to (at least) a stationary point of (10). However, as the global optimum of the problem (10)-(12) is intractable, the gap between the solution in Table I and the global optimum is difficult to obtain.

\section{NumericAl EXAMPLES}

In this section, we study the performance of the proposed joint transceiver matrices design algorithm for interference
TABLE I

Procedure OF SOLVING THE PROBLEM (10)-(12) BY THE PROPOSED ALGORITHM.

1) Initialize the algorithm with $\mathbf{F}^{(0)}$ and $\left\{\mathbf{B}_{k}^{(0)}\right\}$ satisfying (7) and (8); Set $n=0$

2) Obtain $\left\{\mathbf{W}_{k}^{(n+1)}\right\}$ based on (13) with fixed $\mathbf{F}^{(n)}$ and $\left\{\mathbf{B}_{k}^{(n)}\right\}$.

3) Update $\mathbf{F}^{(n+1)}$ through solving the problem (26)-(27) with given $\left\{\mathbf{B}_{k}^{(n)}\right\}$ and $\left\{\mathbf{W}_{k}^{(n+1)}\right\}$.

4) Update $\left\{\mathbf{B}_{k}^{(n+1)}\right\}$ by solving the problem (31)-(33) with fixed $\mathbf{F}^{(n+1)}$ and $\left\{\mathbf{W}_{k}^{(n+1)}\right\}$.

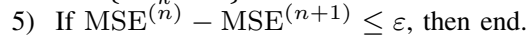
Otherwise, let $n:=n+1$ and go to Step 2 .

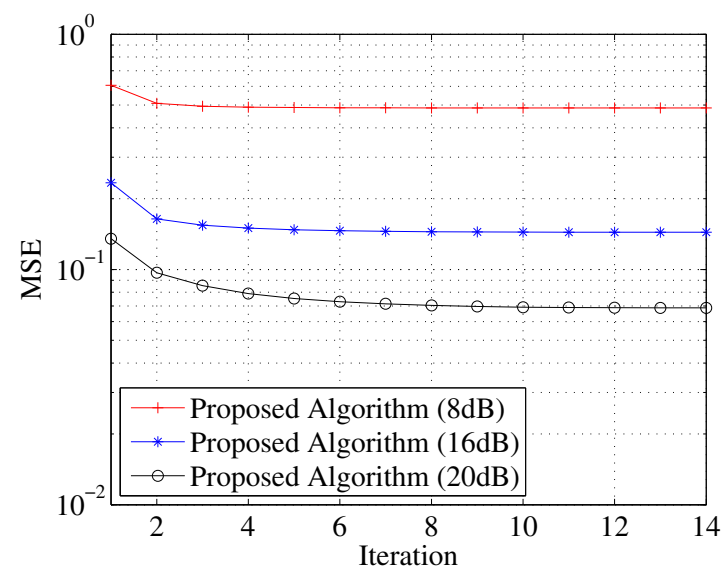

Fig. 2. Example 1: MSE versus the number of iterations, $K=2$.

MIMO relay systems in Table I through numerical simulations. We consider an interference MIMO relay system with $d=3$, where all transmitters and receivers have the same number of antennas, i.e., $N_{s k}=N_{d k}=4, k=1, \cdots, K$, and the relay node has $N_{r}=20$ antennas. We also assume that all transmitters have the same power budget of $P_{s k}=20 \mathrm{~dB}$, $k=1, \cdots, K$. All channel matrices have i.i.d. complex Gaussian entries with zero mean and unit variance, and all noises are i.i.d. Gaussian with zero mean and unit variance. The QPSK constellations are used to modulate the source symbols. All simulation results are averaged over $5 \times 10^{5}$ independent channel realizations. The proposed algorithm is initialized with $\mathbf{F}^{(0)}=\sqrt{P_{r} / \operatorname{tr}\left(\sum_{k=1}^{K} P_{s k} \mathbf{H}_{k} \mathbf{H}_{k}^{H} / N_{s k}+\mathbf{I}_{N_{r}}\right)} \mathbf{I}_{N_{r}}$ and $\mathbf{B}_{k}^{(0)}=\sqrt{P_{s k} / N_{s k}} \mathbf{I}_{N_{s k}}, k=1, \cdots, K$. As a benchmark, the performance of the proposed algorithm is compared with the total leakage minimization (TLM) algorithm developed in [11].

In the first numerical example, we study the convergence speed of the proposed algorithm. Fig. 2 shows the normalized SMSE performance of the proposed algorithm versus the number of iterations at various levels of $P_{r}$ with $K=2$. Interestingly, the convergence speed of the proposed algorithm decreases with increasing $P_{r}$. However, even at $P_{r}=20 \mathrm{~dB}$, the proposed algorithm converges around 10 iterations. In fact, the decreasing of the MSE is negligible after seven iterations. Thus, we suggest that only seven iterations are needed for the 


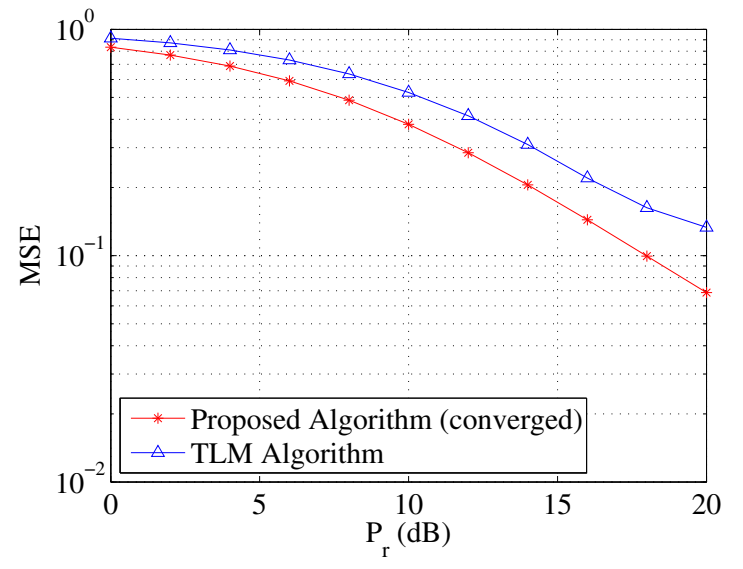

Fig. 3. Example 2: Comparison of MSE versus $P_{r}, K=2$.

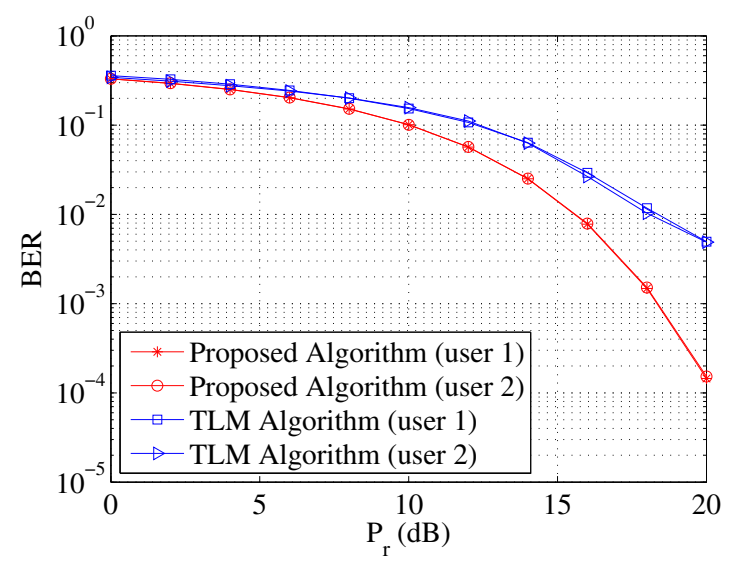

Fig. 4. Example 1: BER versus $P_{r}$ for each transmitter-receiver pair, $K=2$.

proposed algorithm to achieve a good performance.

In the second example, we compare the performance of the proposed algorithm with the TLM algorithm. Fig. 3 shows the normalized SMSE performance of the two algorithms tested versus $P_{r}$ with $K=2$. It can be seen that the proposed algorithm outperforms the TLM algorithm throughout the whole $P_{r}$ range.

For this example, the BER of all transmitter-receiver pairs versus $P_{r}$ yielded by the proposed algorithm is shown in Fig. 4. It can be seen that the proposed algorithm yield smaller BER than the TLM algorithm over the whole $P_{r}$ range. We also observe from Fig. 4 that both transmitter-receiver pairs achieve almost identical BER, indicating that the proposed algorithm is fair to all links.

In the last example, we study the performance of the proposed algorithm with different number of transmitter-receiver pairs $K$. The normalized SMSE performance of the proposed algorithm versus $P_{r}$ is shown in Fig. 5 for $K=2,3,4$. As expected, the MSE increases with $K$.

\section{CONClusion}

We have developed an iterative algorithm for jointly optimizing the transmitter, relay, and receiver matrices of in-

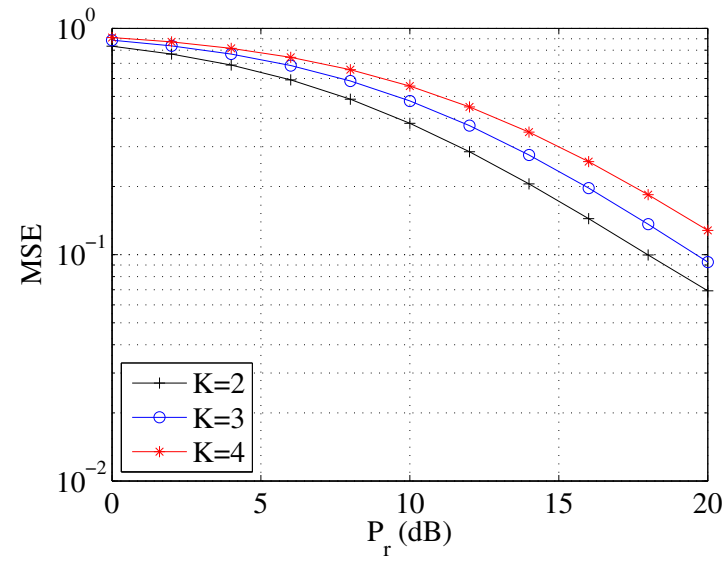

Fig. 5. Example 3: MSE versus $P_{r}$ for different $K$.

terference MIMO relay systems. The optimal structure of the relay precoding matrix has been derived to reduce the computational complexity. Numerical simulation results show that the proposed algorithms has a fast convergence rate.

\section{REFERENCES}

[1] Y. Fan and J. Thompson, "MIMO configurations for relay channels: Theory and practice," IEEE Trans. Wireless Commun., vol. 6, pp. 17741786, May 2007.

[2] L. Sanguinetti, A. A. D'Amico, and Y. Rong, "A tutorial on the optimization of amplify-and-forward MIMO relay systems," IEEE $J$. Selet. Areas Commun., vol. 30, pp. 1331-1346, Sep. 2012.

[3] G. Kramer, M. Gastpar, and P. Gupta, "Cooperative strategies and capacity theorems for relay networks," IEEE Trans. Inf. Theory, vol. 51, pp. 3037-3063, Sep. 2005.

[4] W. Guan and H. Luo, "Joint MMSE transceiver design in nonregenerative MIMO relay systems," IEEE Commun. Lett., vol. 12, pp. 517-519, Jul. 2008.

[5] Y. Rong, X. Tang, and Y. Hua, "A unified framework for optimizing linear nonregenerative multicarrier MIMO relay communication systems," IEEE Trans. Signal Process., vol. 57, pp. 4837-4851, Dec. 2009.

[6] A. Toding, M. R. A. Khandaker, and Y. Rong, "Joint source and relay optimization for parallel MIMO relay networks," EURASIP Journal Advances Signal Process., 2012:174.

[7] M. Maddah-Ali, A. Motahari, and A. Khandani, "Communication over MIMO X channels: Interference alignment, decomposition, and performance analysis," IEEE Trans. Inf. Theory, vol. 54, pp. 3457-3470, Aug. 2008.

[8] B. Nourani, S. Motahari, and A. Khandani, "Relay-aided interference alignment for the quasi-static X channel," in Proc. IEEE Int. Symposium Inf. Theory, Seoul, Korea, Jun. 28-Jul. 3, 2009, pp. 1764-1768.

[9] X. Wang, Y.-P. Zhang, P. Zhang, and X. Ren, "Relay-aided interference alignment for MIMO cellular networks," in Proc. IEEE Int. Symposium Inf. Theory, Cambridge, MA, Jul. 1-6, 2012, pp. 2641-2645.

[10] M. Khandaker and Y. Rong, "Interference MIMO relay channel: Joint power control and transceiver-relay beamforming," IEEE Trans. Signal Process. vol. 60, pp. 6509-6518, Dec. 2012.

[11] K. T. Truong, P. Sartori and R. W. Heath, "Cooperative algorithms for MIMO amplify-and-forward relay networks," IEEE Trans. Signal Process. vol. 61, pp. 1272-1287, Mar. 2013.

[12] Y. Rong, "Linear non-regenerative multicarrier MIMO relay communications based on MMSE criterion," IEEE Trans. Commun., vol. 58, pp. 1918-1923, Jul. 2010.

[13] J. W. Brewer, "Kronecker products and matrix calculus in system theory," IEEE Trans. Circuits Syst., vol. 25, pp. 772-781, Sep. 1978.

[14] S. Boyd and L. Vandenberghe, Convex Optimization. Cambridge, U. K.: Cambridge University Press, 2004.

[15] M. Grant and S. Boyd, "Cvx: Matlab software for disciplined convex programming (webpage and software)," [Online]. Available: http://cvxr.com/cvx, Apr. 2010. 